\title{
Enteritis eosinofílica, una causa extraña de abdomen agudo. reporte de caso clínico
}

\author{
Eosinophilic enteritis, a strange cause of acute abdomen \\ Clinical case report \\ Salamea Avilés Pablo Andrés', Salamea Avilés Juan Josué², \\ Salamea Cordero Román Marcelo ${ }^{1}$
}

VOLUMEN 38 | N 2 | AGOSTO 2020

FECHA DE RECEPCIÓN: 21/05/2020 FECHA PUBLICACIÓN: 31/08/2020 FECHA DE REVISIÓN: 18/01/2021 FECHA DE REEDICIÓN: 15/03/2021

1. Libre ejercicio. Cuenca - Ecuador

2. Libre ejercicio. Guayaquil Ecuador

\begin{tabular}{c|c} 
Caso & $\begin{array}{c}\text { Clinical } \\
\text { Case }\end{array}$ \\
\hline
\end{tabular}

DOI: https://doi.org/10.18537/RFCM.38.02.04

Correspondencia:

pabloandressalamea@gmail.com

Dirección:

José Joaquín de Olmedo y Nuñez de Bonilla

Código Postal:

010104

Teléfono:

0999856672

Cuenca - Ecuador

\section{RESUMEN}

La enteritis eosinofílica, es una patología rara, caracterizada por infiltración de eosinófilos en una o más capas histológicas en diferentes niveles del tracto gastrointestinal, siendo el estómago e intestino delgado los más afectados; su cuadro clínico, inespecífico, caracterizado por dolor abdominal, náusea, vómito, diarrea o estreñimiento, pérdida de peso y ascitis, con presencia o ausencia de eosinofilia en la biometría.

\section{Reporte de caso}

Paciente masculino de 51 años de edad, acudió a emergencia por distensión abdominal y náusea, al examen físico en la palpación intenso dolor y distención abdominal, percusión timpánico y abolición de ruidos hidroaéreos. La analítica reportó leucocitosis con neutrofilia, radiografía de abdomen íleo adinámico, en la ecografía abdominal presencia de imágenes tubulares con aspecto de diana, asociado a líquido libre purulento en fosa ilíaca derecha y fondo de saco vésico rectal. Un cuadro clínico compatible con abdomen agudo de resolución quirúrgica, se realizó laparotomía exploratoria.

Palabras clave: eosinofilia, abdomen agudo, enteritis.

\section{ABSTRACT}

The eosinophilic enteritis is a rare pathology, characterized by infiltration of eosinophils in one or more histological layers at different levels of the gastrointestinal tract, the stomach and small intestine being the most affected; its nonspecific clinical picture, characterized by abdominal pain, nausea, vomiting, diarrhea or constipation, weight loss and ascites, with the presence or absence of eosinophilia in the biometry. 


\section{Case report}

A 51-year-old male patient came to the emergency room due to abdominal distention and nausea, to physical examination on palpation, intense abdominal pain and distention, tympanic percussion and abolition of air-fluid sounds. Laboratory analysis reported leukocytosis with neutrophilia, abdominal ileus adynamic radiography, abdominal ultrasound presence of tubular images with a target appearance, associated with free purulent fluid in the right iliac fossa and rectal vesicum fundus. A clinical picture compatible with an acute abdomen with surgical resolution, an exploratory laparotomy was performed.

Key words: eosinophilia, abdomen acute, enteritis.

\section{INTRODUCCIÓN}

Descrita por primera vez en 1937 por Kaijser en 2 pacientes, y desde entonces, hasta 2008 se han reportado aproximadamente 300 casos $[1,2]$, la causa y patogénesis de la enteritis eosinofílica aún no están claros, se conoce que hasta el $75 \%$ de los pacientes presentan alergias a medicamentos, alimentos o enfermedades atópicas como el asma [1-3].

Las alteraciones eosinofílicas en el tubo digestivo son raras e infrecuentes, y se caracterizan por infiltración en las distintas capas intestinales siendo el estómago y el intestino delgado las regiones anatómicas más comprometidas. Su sintomatología es variable e inespecífica dependiendo de la capa histológica afectada, el cuadro clínico se determina por la presencia de dolor abdominal, náuseas, vómitos, saciedad precoz, diarrea, pérdida de peso, ascitis, más la presencia o no de eosinofilia periférica y antecedentes de alergia o intolerancia a algunos alimentos [1-
4]. El diagnóstico se basa en los infiltrados eosinofílicos en el tracto gastrointestinal, los resultados de patología del segmento resecado y los resultados del líquido ascítico enviado a estudio, así como la ausencia de eosinofilia en otros órganos [2-6].

Siendo la enteritis eosinofílica una patología rara, con manifestaciones clínicas inespecíficas, que evoluciona a un abdomen agudo, y tratándose de un caso reportado por primera vez en el sur del Ecuador, es de importancia hacer el presente reporte.

\section{CASO CLÍNICO}

Paciente de 51 años, de sexo masculino, que acudió al departamento de emergencias de uno de los principales hospitales públicos de la ciudad de Cuenca por presentar dolor abdominal tipo cólico de 6 días de evolución, indicó que durante ese lapso de tiempo se automedicó analgésicos y antiespasmódicos sin presentar mejoría, al cuadro clínico se sumó distensión abdominal y sensación de náusea.

Como antecedentes patológicos importantes, se deben citar el diagnóstico de asma bronquial de larga data (15 años), en tratamiento intermitente con salbutamol.

Al examen físico, abdomen distendido y a la auscultación ruidos hidroaéreos abolidos y tintineo metálico, a la percusión timpanismo y a la palpación superficial y profunda se encontró intenso dolor abdominal con signo de blumberg positivo; y posteriormente evolucionando a irritación peritoneal generalizado.

\section{EXÁMENES COMPLEMENTARIOS}

Hemograma 


\begin{tabular}{lccc}
\hline \multicolumn{1}{c}{ Parámetro } & Resultado & Unidad & Valor Referencial \\
\hline Leucocitos & 29.020 & $\mathrm{~mm} 3$ & $5000-10000 \mathrm{~mm} 3$ \\
Neutrófilos & 85 & $\%$ & $45-70 \%$ \\
Linfocitos & 9 & $\%$ & $20-40 \%$ \\
Monocitos & 3 & $\%$ & $1-6 \%$ \\
Eosinófilos & 8 & $\%$ & $0-6 \%$ \\
Basófilos & 0 & $\%$ & $0-1 \%$ \\
Hemoglobina & 13.4 & $\mathrm{~g} / \mathrm{dl}$ & $12-16 \mathrm{~g} / \mathrm{dl}$ \\
Hematocrito & 42.6 & $\%$ & $37.5-48 \%$ \\
Plaquetas & 315.000 & $10 \star 3 \mathrm{uL}$ & $150-40010 \star 3 \mathrm{uL}$ \\
Urea & 21.5 & $\mathrm{mg} / \mathrm{dl}$ & $10-50 \mathrm{mg} / \mathrm{dl}$ \\
Creatinina & 1.21 & $\mathrm{mg} / \mathrm{dl}$ & $0.5-0.9 \mathrm{mg} / \mathrm{dl}$ \\
\hline
\end{tabular}

Los parámetros que se encontraron en la biometría hemática reflejan leucocitosis marcada más neutrofilia, lo cual orienta a pensar en reacción inflamatoria, que sumado a la sintomatología lleva a pensar en un abdomen agudo de tipo quirúrgico; además, llamando la atención la hipereosinofilia, pues generalmente se presenta en cuadros de alergias y en infecciones parasitarias intestinales.

\section{Imagen $\mathrm{N}^{\circ} 1$}

Rx abdomen de pie

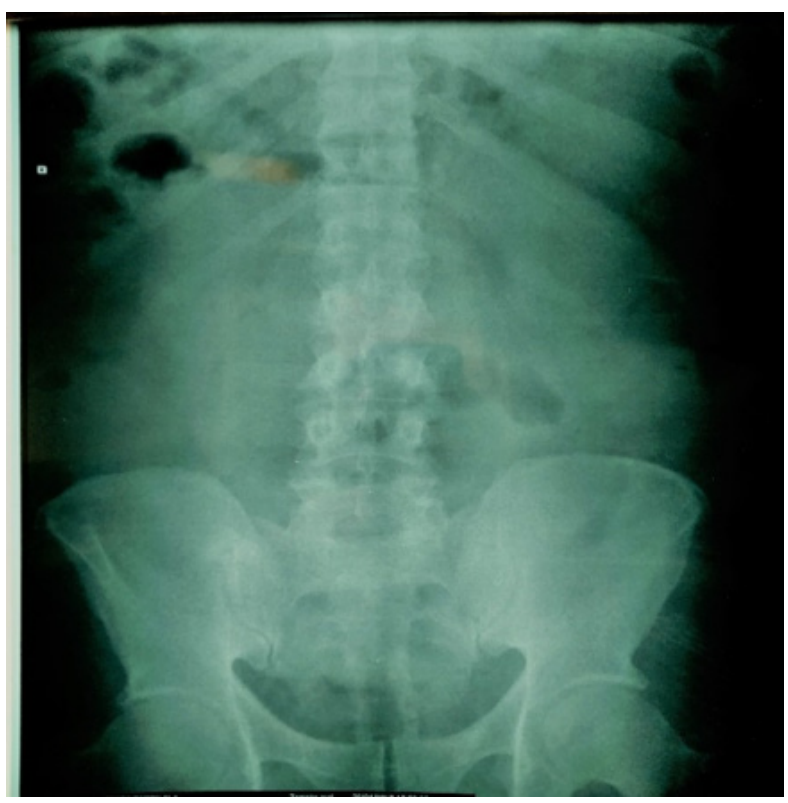

En fosa ilíaca derecha se observa radioopacidad, presencia de íleo a nivel de mesogástrico, con formación de niveles hidroaéreos, gas en ampolla rectal y opacidad de las sombras de los músculos ilíacos.

Imagen $\mathrm{N}^{\circ} 2$

Ecografía abdominal

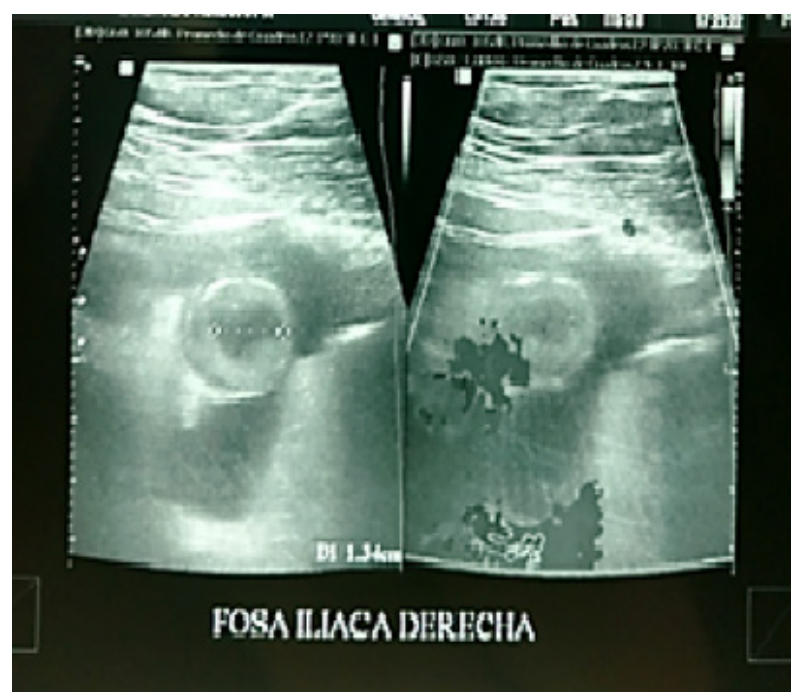

En fosa ilíaca derecha se observan imágenes tubulares con aspecto de diana que se encuentran rodeadas de contenido anecoide compatible con líquido libre con volumen aproximado 20-30cc en fondo de saco, se presentan signos de un proceso inflamatorio.

\section{TRATAMIENTO}

Serealizó unalaparotomía exploratoria en donde se identificaron asas distendidas, en el íleon a $80 \mathrm{~cm}$ de la válvula ileocecal se presentaron 
cambios en la coloración con presencia de punteado equimótico, ciertas zonas de color violáceas - negruzcas sin motilidad intestinal (Imagen $\mathrm{N}^{\circ} 3$ y $\mathrm{N}^{\circ} 4$ ). Además, abundante líquido libre serosanguinolento en cavidad, por lo que se toma muestra y es enviado para análisis. Se resecó aproximadamente $10 \mathrm{~cm}$ de íleon más la inmediata anastomosis término terminal (el intestino resecado es enviado a patología para análisis. Imagen $\mathrm{N}^{\circ}$ 5). Posteriormente se procedió con el cierre de la cavidad abdominal por planos y pasa a sala de recuperación. Paciente presentó evolución favorable durante los 5 días de permanencia hospitalaria, luego de lo cual recibe alta médica. En el control a los 10 días se evidenció una evolución clínica favorable con el retiro de puntos de sutura de piel, fue revalorado a los 30 días con buen proceso de cicatrización, abdomen sin patología aparente y paciente en buen estado general.

\section{Imagen $\mathrm{N}^{\circ} 3$}

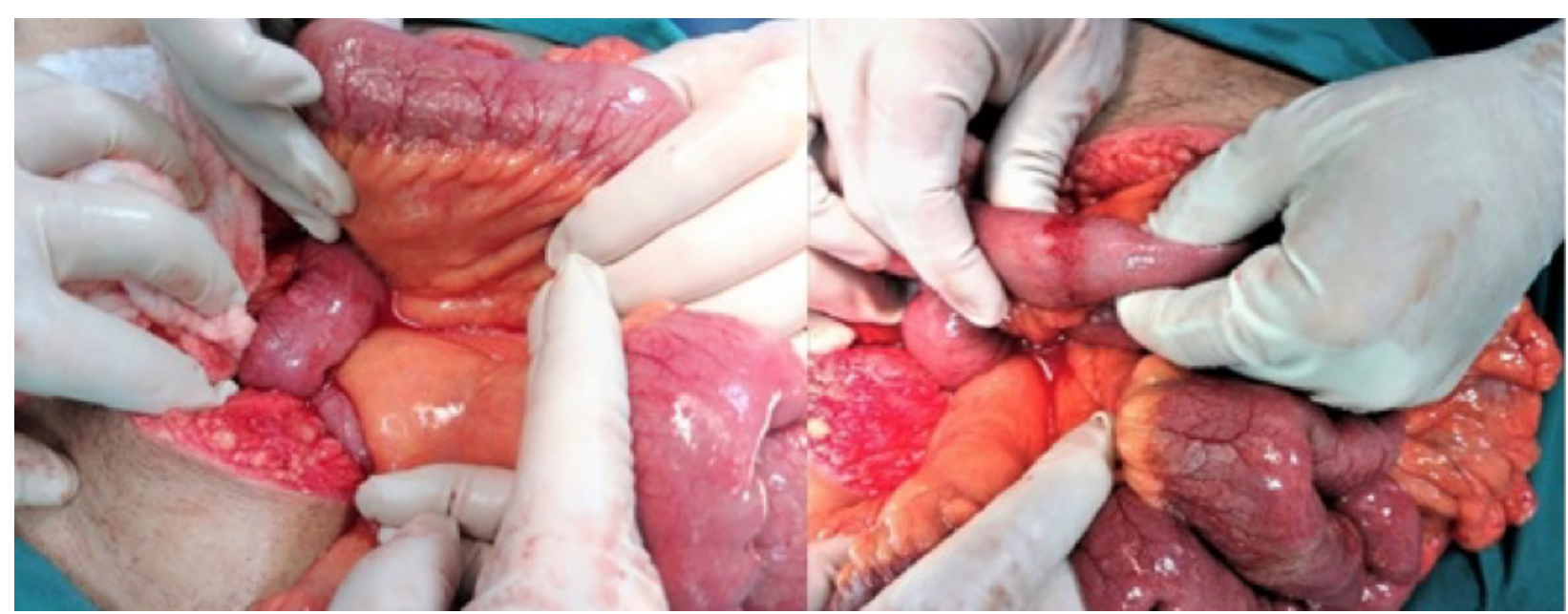

Se observa presencia de líquido libre serosanguinolento. Se identifica asas intestinales distendidas, punteado equimótico y cambios de coloración en la pared intestinal.

\section{Imagen $\mathrm{N}^{\circ} 4$}

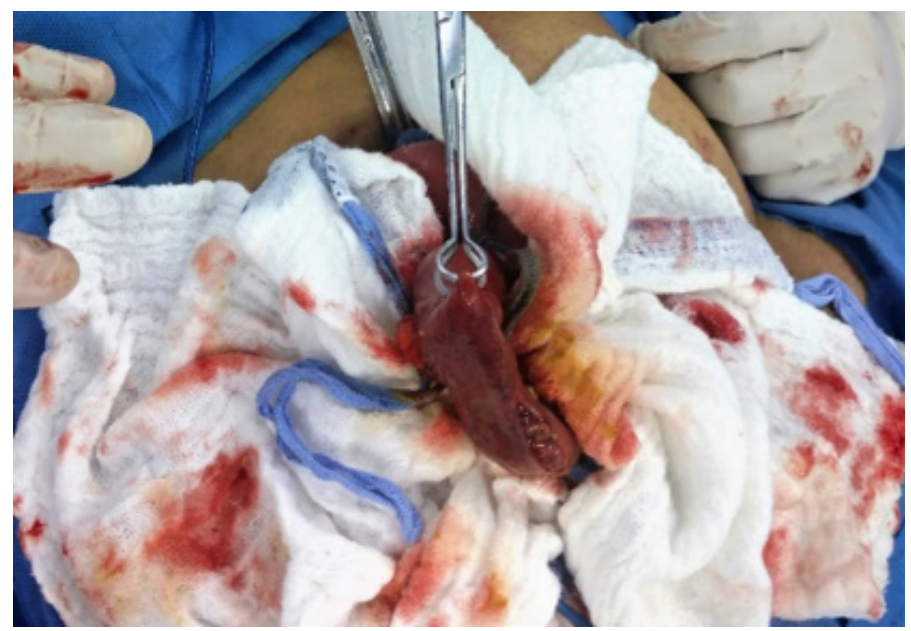

Segmento intestinal violáceo, sin motilidad. 


\section{Imagen $\mathrm{N}^{\circ} 5$}

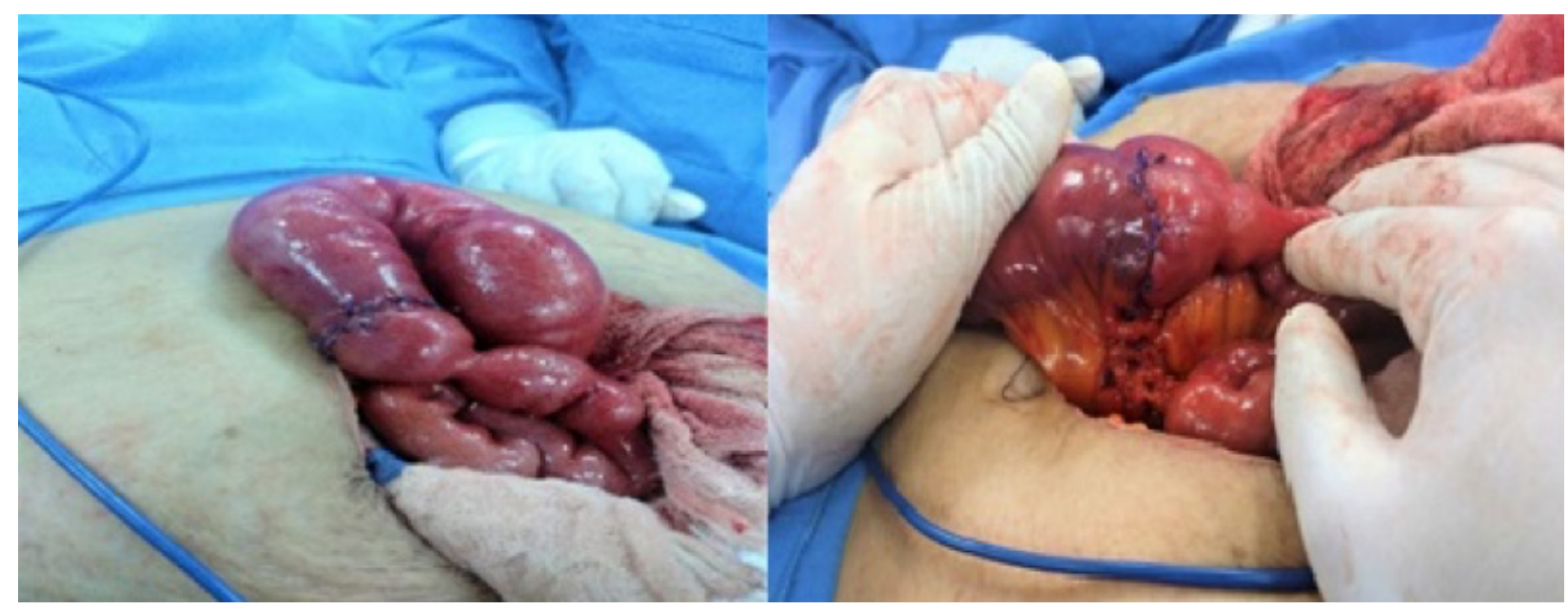

Resección intestinal de aproximadamente $10 \mathrm{~cm}$ más anastomosis término terminal.

Resultado de anatomía patológica: indica que el segmento de intestino delgado (íleon) receptado, presenta macroscópicamente cambio de coloración de la normalidad y microscópicamente se puede observar infiltración por células de tipo polimorfonucleares eosinófilos maduros en serosa y capa muscular.

Análisis bioquímico de líquido peritoneal: líquido de aspecto serosanguinolento, leucocitos < 50/ mm3, eosinófilos > 100/mm3.

\section{DISCUSIÓN}

La enteritis eosinofílica es una patología rara que comúnmente afecta estómago e intestino delgado [1-3], el segmento intestinal afectado en este caso fue el íleon.

La analítica sanguínea puede presentarse con o sin eosinofilia, por lo tanto, se debe ahondar en el hecho que la elevación de los mismos no son un criterio diagnóstico como lo mencionan Cebollero - Fuentes [3] y Gómez - Morales [4], los valores del paciente del caso presentado reflejan un incremento sustancial del porcentaje de eosinófilos, lo cual orienta a pensar en una reacción alérgica o una infestación masiva de parásitos a nivel intestinal $[7,8]$.

Los antecedentes patológicos en estos casos, revisten gran importancia, ya que hasta el 75\% de los pacientes que cursan con esta patología son alérgicos a medicamentos, alimentos o tienen como diagnóstico una enfermedad atópica o inmunológica [1]; en la serie de casos clínicos de Álvarez - Sánchez [9] se concuerda con el caso materia de esta presentación, el mismo que tiene como diagnóstico desde hace más de 15 años asma bronquial bajo tratamiento intermitente con salbutamol.

Según Bonilla [1] las manifestaciones clínicas dependen de la extensión histológica que abarca el proceso, por ello, es de vital importancia el reporte de anatomía patológica para un acertado diagnóstico, lo cual coincide con este caso, el mismo que reporta infiltración a nivel de capa serosa y capa muscular $[1,10]$. Belenguer - Aguilar [5] y Salazar [6] indican que la afectación en la capa serosa del intestino causa ascitis y en la capa muscular produce náusea, dolor abdominal, obstrucción intestinal, pudiendo inclusive emular un cuadro de apendicitis aguda si se ve afectado el íleon [1-11], y manejarse probablemente con un diagnóstico de obstrucción intestinal o aún 
más, simplificar su importancia y tratar con un diagnóstico genérico de "abdomen agudo."

Como diagnóstico diferencial de la enteritis eosinofílica se encuentra la enfermedad de Crohn y la colitis ulcerosa, ya que ambas presentan infiltración eosinofílica en la pared intestinal, clínicamente solo la enfermedad de Crohn se comporta de forma similar tanto en presencia de dolor y distensión abdominal marcada [4-12]. El criterio clínico de la colitis ulcerosa varía, pudiendo llegar a presentar deposiciones diarreicas de tipo sanguinolento y manifestaciones extraintestinales, también en el análisis histológico se observan inflamación de la mucosa, úlceras superficiales y micro abscesos [13-14]; por ello se realza la importancia que el análisis histológico tiene, para poder llegar a un diagnóstico definitivo.

El estudio de imagen utilizado en la serie de casos de Gómez - Morales [4] fue la TAC abdominal la misma que reportó líquido libre más asas intestinales distendidas, mientras que, en el caso presentado, por motivo de accesibilidad y disponibilidad se realizó además de una radiografía de abdomen, una ecografía abdominal, la misma que reporta asas distendidas y líquido libre en cavidad abdominal; se acota que los estudios imagenológicos son solamente una herramienta de apoyo diagnóstico, pues un adecuado examen físico y una buena orientación clínica permitirán tomar decisiones acertadas.

Es de mucho valor lo presentado en sus respectivos estudios tanto Amores Arredondo [8] como Álvarez - Sánchez [9], ya que la sospecha diagnóstica de la enteritis eosinofílica es transquirúrgica, pero para obtener un diagnóstico definitivo debe apoyarse en los resultados que la anatomía patológica y el laboratorio proporcionen. En el caso presentado, anatomía patológica reporta infiltrado eosinofílico en varias capas del intestino delgado y laboratorio indica un valor elevado de eosinófilos en el líquido peritoneal $[9,13]$, se apoya en las imágenes que se disponen de este caso, y se puede valorar un cambio macroscópico de coloración en la pared intestinal que llevó a la resección quirúrgica de dicho segmento intestinal.

\section{CONCLUSIÓN}

La enteritis eosinofílica es una patología poco común y muy poco reportada, de hecho, en el Ecuador no existe ningún estudio ni caso clínico sobre el tema y son muy pocos los casos reportados en América Latina.

El cuadro clínico de la enteritis eosinofílica es variable y obviamente dependiendo de la clínica podrá ser manejado como un abdomen agudo quirúrgico o no quirúrgico.

Los hallazgos quirúrgicos y el resultado proporcionado por anatomía patológica del segmento intestinal resecado y los valores que el laboratorio facilite sobre el líquido peritoneal obtenido, nos ofrecen el diagnóstico de esta patología.

La evolución clínica de estos pacientes es favorable sin que exista generalmente reincidencia en la presentación del mismo cuadro clínico, no siempre el manejo será quirúrgico.

\section{PERSPECTIVA DEL PACIENTE}

Paciente refiere que el tratamiento que se realizó mejoró la sintomatología que presentaba al ingreso y que su evolución a los cinco días de su tratamiento fue favorable; además que al pasar los días ya no se presentaba dolores abdominales y que cada vez se sentía de una mejor manera hasta la última consulta médica que recibió a los treinta días del procedimiento 
quirúrgico. Se sentía agradecido por el tratamiento y los cuidados brindados para su recuperación por el personal médico.

\section{ASPECTOS BIOÉTICOS}

El presente trabajo cuenta con el consentimiento informado del paciente y el manejo confidencialidad de los datos.

\section{INFORMACIÓN DE LOS AUTORES}

Salamea Avilés Pablo Andrés. Médico. Libre ejercicio. Maestrante de la Universidad Espíritu Santo. Cuenca-Azuay-Ecuador.

e-mail: pabloandressalamea@gmail.com ORCID:https://orcid.org/0000-0001-7750-3288

Salamea Avilés Juan Josué. Médico. Especialista en Cirugía General. Libre ejercicio. Guayaquil-Guayas-Ecuador.

e-mail: juansalamea@hotmail.com

ORCID:https://orcid.org/0000-0002-9030-8951

Salamea Cordero Román Marcelo. Doctor en Medicina y Cirugía. Libre Ejercicio. CuencaAzuay-Ecuador

e-mail: romarsalamea@hotmail.com

ORCID:https://orcid.org/0000-0002-7495-0450

\section{CONTRIBUCIÓN DE LOS AUTORES}

Los autores declaran haber participado en la concepción y diseño del trabajo, análisis e interpretación de los datos, redacción y revisión crítica del manuscrito, y aprobación de la versión final.

\section{CONFLICTO DE INTERESES}

No existió conflicto de intereses durante el desarrollo del artículo.

\section{FUENTES DE FINANCIAMIENTO}

Autofinanciado

\section{REFERENCIAS BIBLIOGRÁFICAS}

1. Roberto $S$, Bonilla R, Pérez G. Gastroenteropatía Eosinofílica, Reporte de un Caso y Revisión de la Literatura Rev Colomb Gastroenterol [Internet]. 30 de diciembre de 2014; [citado 12 mayo 2017]; 29(4):419-23. Disponible en: https:// www.revistagastrocol.com/index.php/rcg/ article/view/439

2. Arellano G, Ramírez F, Aguirre J, Domínguez L. Gastroenteritis Eosinofílica. Medigraphic [Internet]; 2015 [citado 10 de mayo 2017] ;(4):6. Disponible en: http://www. medigraphic.com/pdfs/actmed/am-2015/ am154e.pdf

3. Cebollero Benito M. P., Fuentes Solsona F., Escartín Valderrama J., Valenzuela Martínez M. J., Ibáñez Mirallas J. P., Esarte Muniáin J. Enteritis Eosinofílica como causa de Abdomen Agudo. An Med. Interna (Madrid) [Internet]. 2002 Mayo [citado 2017 mayo 10]; 19 (5): 31-32. Disponible en: http:// scielo.isciii.es/scielo.php?script =sci_ arttext\&pid=S0212-71992002000500007

4. Gómez J, Morales D. Gastroenteritis Eosinofílica. Trujillo: Rev. Soc. Peruana Med. Interna [Internet]; 2012 [citado 10 de mayo 2017]; 23(4):5. Disponible en: http:// sisbib.unmsm.edu.pe/BvRevistas/spmi/ v23n4/pdf/a09v23n4.pdf

5. Belenguer L, Aguilar E, Cucurella L, Cay A. Enteritis Eosinofílica como causa de Abdomen Agudo. Teruel: Anales de Medicina Interna; 2010 [citado 10 de Mayo 2017]. Disponible en: http://scielo.isciii.es/ pdf/ami/v21n11/carta1.pdf

6. Salazar F. Gastroenteritis Eosinofílica. Lima: Rev Gastro Perú [Internet]; 1995 [citado 11 de mayo 2017]; 15(2). Disponible en: http:// 
sisbib.unmsm.edu.pe/bVrevistas/gastro/ Vol_15N2/gastroenteritis_eosinofilica.htm

7. Townsend M., Beauchamp R., Evers B., Mattox K. Sabiston Cirugía General y del Aparato Digestivo. 19a ed. Barcelona: Elsevier; 2014.

8. Amores J., Arredondo A., Ávila D., Reyes R. Enteritis Eosinofílica Primaria: a propósito de un caso. AMC [Internet]. 2009 Jun [citado 11 de mayo 2017]; 13(3). Disponible en: http://scielo.sld.cu/ scielo.php?script=sci_arttext\&pid=S102502552009000300017\&lng=es.

9. Álvarez García J.F., Sánchez Sánchez M.T., Chiquero Palomo M., Costo Campoamor A., Saponi Cortés J. M., Pérez Reyes F. et al. Gastroenteritis Eosinofílica: a propósito de dos casos clínicos. Cáceres: An. Med. Interna (Madrid) [Internet]. 2004 Sep [citado 11 de mayo 2017]; 21 (9): 45-49. Disponible en: http:// scielo.isciii.es/scielo.php?script $=$ sci $_{-}$ arttext\&pid=S0212-71992004000900007

10. Garibay O, Soto D, Coria E, Castrejón M, Vargas M, Ramos $G$ et al. Gastroenteritis Eosinofílica. Ciudad de México: Revista Alergia México. 2014. [citado 14 de mayo 2017]. Disponible en: nietoeditores. com.mx/nieto/Alergia/2014/jul-sep/ Alergia3small.pdf

11. Arredondo A, Heredia A, Mejías J, Rivero E. Enteritis eosinofílica. A Propósito de dos casos. Rev. Med. Electrón. [Internet]. 2014 Jun [citado 11 de mayo 2017]: 36(3): 350-357. Disponible en: http:// scielo.sld.cu/scielo.php?script $=$ sci_ arttext\&pid=S1684-18242014000300011

12. Peters J, Matthews J, McFadden D, Pemberton J. Cirugía del Tracto Alimentario de Shackelford. 7ma ed. New York: Amolca; 2016.

13. Aláez-Chillarón A, Pérez E, Kilmurray $L$, Martín F, Mojtar F, Moreno I. Enteritis Eosinofílica, una Causa Poco Frecuente de Intervención Quirúrgica Urgente. Gastroenterol Hepatol [Internet]. 1 de junio de2014 [citado11 demayo2017];37(6):3546.. Disponible en: http://www.elsevier.es/ es-revista-gastroenterologia-hepatologia14-articulo-enteritis-eosinofilica-unacausa-poco-S0210570513002628

14. Ricart E. Colitis Ulcerosa. Barcelona: Gastroenterología y Hepatología. 2012. [acceso 20 de octubre 2017]. Disponible en: http://www.aegastro.es/sites/default/ files/archivos/ayudas-practicas/32_ Colitis_ulcerosa.pdf 